\title{
ANÁLISE ACÚSTICA DA VOZ DE MULHERES IDOSAS
}

\author{
Voice acoustic analysis in elderly women
}

\author{
Janaína da Silva Berto Cerceau (1), Cláudia Fernanda Tolentino Alves ${ }^{(2)}$, Ana Cristina Côrtes Gama ${ }^{(3)}$
}

\begin{abstract}
RESUMO
Objetivo: avaliar medidas acústicas da voz de idosas. Métodos: participaram 96 idosas, com idades entre 60 e 103 anos, divididas em três grupos conforme a faixa etária. Resultados: valores da frequência fundamental foram: 193,81 Hz (Grupo 1), 195,71 (Grupo 2) e 187,60 Hz (Grupo 3). Extensão fonatória: 42,10 semitons (Grupo 1); 37,29 semitons (Grupo 2) e 38,75 semitons (Grupo 3). Os valores da frequência mínima e máxima foram, respectivamente, 103,62 Hz e 1099,1 Hz (Grupo 1); $102,28 \mathrm{~Hz}$ e $918,10 \mathrm{~Hz}$ (Grupo 2) e $88,73 \mathrm{~Hz}$ e $876,8 \mathrm{~Hz}$ (Grupo 3). Os valores do Tempo Máximo de Fonação da vogal /a/ foram 8,54 segundos (Grupo 1), 7,97 segundos (Grupo 2) e 7,52 segundos (Grupo 3). Para a vogal /i/ os valores foram de 8,82 segundos (Grupo 1), 8,44 segundos (Grupo 2) e 8,05 segundos (Grupo 3). Para a vogal /u/ os valores foram de 9,22 segundos (Grupo 1), 8,09 segundos (Grupo 2) e 7,95 segundos (Grupo 3). Conclusão: mulheres idosas apresentam uma frequência fundamental mais grave e tempos máximos de fonação das vogais diminuídos. A extensão fonatória máxima apresentou valores semelhantes aos de adultos jovens. Todas as medidas estudadas mostraram uma tendência de diminuição com o avanço da idade.
\end{abstract}

DESCRITORES: Voz; Envelhecimento; Acústica da Fala

\section{INTRODUÇÃO}

O aumento da longevidade e a redução das taxas de mortalidade, nas últimas décadas do século passado, mudaram o perfil demográfico do Brasil ${ }^{1}$. Fazse necessário investigar sobre a saúde na terceira idade, pois rapidamente este país deixa de ser "de jovens" e o envelhecimento torna-se questão fundamental para as políticas públicas ${ }^{2}$. Os brasileiros com mais de 60 anos representam $8,6 \%$ da população e esta proporção chegará a 14\% em 2025, o que representa 32 milhões de idosos ${ }^{1}$.

Até os 25 anos, aproximadamente, o ser humano é submetido ao processo de desenvolvimento, por meio do qual atinge o ápice de suas funções. O objetivo desse processo é garantir a perpetuação da espécie de maneira mais eficiente possível. A partir daí, nos anos que se seguem, o processo de desenvolvimento dá lugar ao processo de envelhecimento, uma série de alterações que têm início em

\footnotetext{
(1) Fonoaudióloga.

(2) Fonoaudióloga.

(3) Fonoaudióloga; Professora Adjunto do Departamento de Fonoaudiologia da Universidade Federal de Minas Gerais, UFMG, Belo Horizonte, MG; Doutora em Ciências dos Distúrbios da Comunicação pela Universidade Federal de São Paulo.
}

torno dos 25-30 anos e vão ganhando mais velocidade a partir dos 40 anos ${ }^{2}$.

O envelhecimento é um processo progressivo e degenerativo, caracterizado pela redução da eficiência funcional, com enfraquecimento dos mecanismos de defesa frente às variações ambientais e perda das reservas funcionais. Além disso, universal entre as espécies e intrínseco, ou seja, não é determinado por fatores ambientais, apesar de ser influenciado por eles e distingue-se das doenças e patologias que são muitas vezes reversíveis e não observadas igualmente em todas as pessoas ${ }^{3,4}$.

Com o envelhecimento ocorrem modificações no mecanismo da voz e a Fonoaudiologia tem como um de seus objetivos promover a comunicação, lentificando o processo de deterioração vocal ${ }^{5}$. A voz presbifônica (prebys, do grego $=$ homem velho; phoneo, do grego = vocalizar ou emitir sons) caracteriza-se pela redução significativa do tempo máximo de fonação e da extensão fonatória máxima para ambos os sexos. Os homens passam a apresentar uma frequência fundamental mais aguda, e, as mulheres, por sua vez, adquirem uma voz mais grave ${ }^{3}$.

A avaliação da voz de um indivíduo correlaciona dados auditivos, visuais e acústicos para se desenvolver a base de um raciocínio clínico diagnóstico e 
terapêutico. A avaliação perceptivo-auditiva é subjetiva e possibilita a obtenção de aspectos culturais e emocionais do sujeito, além do possível impacto social da disfonia. Já a estroboscopia permite uma visualização da anatomia e fisiologia da região laríngea, enquanto a avaliação acústica realiza mensurações objetivas do sinal sonoro vocal. Para uso clínico, são medidas acústicas importantes a frequência fundamental, o perfil da extensão vocal e o tempo máximo de fonação, dentre outras ${ }^{3,6}$.

A frequência fundamental $\left(f_{0}\right)$ é a velocidade na qual uma forma de onda se repete por unidade de tempo, o que é indicado por $\mathrm{Hz}$ (Hertz). $\mathrm{A}_{0}$ da voz de um indivíduo é o reflexo das características biodinâmicas das pregas vocais e de sua integração com a pressão subglótica, sendo afetada pelo sexo e pela idade ${ }^{3}$.

A extensão fonatória máxima representa a faixa de variação de frequências que um indivíduo consegue emitir, da mais grave a mais aguda, não importando a qualidade vocal conseguida. Esta medida depende da constituição de cada aparelho fonador e tem relação direta com a saúde das pregas vocais.

O valor do Tempo Máximo de Fonação (TMF) é obtido pela medida do tempo máximo que um indivíduo consegue sustentar uma emissão de um som ou de fala encadeada, numa só expiração, e permite uma investigação qualitativa e quantitativa da fonação. Essa medida tem sua importância, pois representa o número de recargas aéreas realizadas a cada um terço de seu tempo máximo de fonação, durante fala encadeada ${ }^{3}$.

Conhecer o processo normal de envelhecimento é fundamental para atuar com prevenção e assim, elaborar programas de reabilitação vocal eficientes. Portanto, o objetivo do presente trabalho foi avaliar medidas acústicas da voz de idosas, analisando a frequência fundamental e as frequências mínima e máxima em Hertz, a extensão fonatória máxima em semitons e o tempo máximo de fonação em segundos da voz de mulheres idosas e compará-las em diferentes faixas etárias.

\section{MÉTODOS}

A população que participou deste estudo foi composta por 96 idosos, do sexo feminino, com idades variando entre 60 a 103 anos. Os participantes foram recrutados em centros de convivência na cidade de Belo Horizonte. Os critérios de inclusão dos sujeitos neste trabalho foram: idade igual ou superior a 60 anos, ausência de comprometimento cognitivo, de queixa vocal, de lesão laríngea e/ou de realização de cirurgias de lesão laríngea.
Para se determinar o tamanho da população de idosas que participaram desta pesquisa, utilizou-se um erro de estimativa de $10 \%$ e um nível de significância de 95\%, com um valor total de 96 idosas.

As participantes foram distribuídas em três grupos, conforme a faixa etária, a saber:

a) Grupo 1: constituído por 51 idosas de 60 a 69 anos:

b) Grupo 2: constituído por 28 idosas de 70 a 79 anos:

c) Grupo 3: constituído por 17 idosas com idade acima de 80 anos.

Houve certo predomínio de indivíduos no primeiro grupo, o que era de se esperar em função da faixa etária deste grupo ser menor.

Foi realizado um estudo observacional com corte transversal. As idosas foram convidadas a participar deste estudo e orientadas sobre os procedimentos aos quais seriam submetidas. Receberam informações orais e escritas (carta de informação) sobre o objetivo do trabalho assim como, de todos os aspectos éticos que regem as pesquisas com seres humanos. Todas as participantes concordaram com a realização da pesquisa e a divulgação dos resultados obtidos por meio da assinatura do Termo de Consentimento Livre e Esclarecido.

Inicialmente foi realizado um questionário com o objetivo de selecionar os indivíduos participantes da amostra, abordando os seguintes aspectos: identificação do sujeito (nome, idade, sexo, profissão), queixa (em relação à voz), alterações vocais e tratamentos realizados (Figura 1). É interessante ressaltar que foram realizadas 103 entrevistas e deste total, sete sujeitos foram excluídos.

As causas da exclusão foram: diagnóstico de nódulo vocal (3); diagnóstico de nódulo vocal e Edema de Reinke (1); cirurgia laríngea para a retirada de nódulo (1), dificuldade cognitiva (2).

As vozes foram gravadas, individualmente, em um ambiente silencioso, com os sujeitos assentados. Solicitou-se a emissão sustentada das vogais /a/, /i/ e /u/ na frequência e intensidade habituais, ou seja, o mais natural e longo possível, em três emissões consecutivas. Os valores dos TMF foram medidos por meio do auxílio de um cronômetro digital marca Cassio ${ }^{\circledR}$, tendo como resultado final o valor médio das três emissões.

Instruiu-se a emissão da vogal /a/ do mais grave ao mais agudo possível, de maneira confortável por três vezes consecutivas, para obtenção da medida da extensão fonatória máxima em semitons e dos valores de frequências máximas e mínimas em $\mathrm{Hz}$. Considerou-se como resultado de tais medidas o valor médio das três emissões.

A $f_{0}$ foi extraída da melhor emissão, dentre as três emissões da vogal /a/ sustentada. O tempo de 


\section{Identificação pessoal:}

Nome:

Idade:

Telefone:

\section{Anamnese:}

1. A senhora acha que a sua voz mudou com a idade?

( ) $\operatorname{sim}($ ) não

2. A senhora tem dificuldade para alcançar algum tom (mais grave ou mais agudo)

devido à idade?

( ) sim ( ) não Qual?

3. Qual a sua atividade profissional?

( ) se aposentada Qual atividade exercia?

( ) se em exercício Qual atividade exerce?

( ) do lar

4. A senhora percebe cansaço ou mudança na voz há mais de quinze dias?

( ) $\operatorname{sim}($ ) não Qual?

5. A senhora já fez alguma avaliação otorrinolaringológica?

( ) $\operatorname{sim}($ )não

6. Caso tenha feito, qual foi o diagnóstico?

7. A senhora acha que, devido à idade, a sua voz está:

( ) sem alteração ( ) mais fina ( ) mais grossa ( ) mais rouca ( ) mais fraca

( ) se outro não descrito acima, qual?

Figura 1 - Protocolo utilizado para caracterização sócio-demográfica e levantamento de sintomas vocais e laríngeos dos participantes

emissão analisado acusticamente foi de, em média, 5,32 ms milissegundos (desvio padrão 0,76).

$O$ registro das vozes foi realizado em um gravador profissional com fita DAT (Digital Áudio Tapes) acoplado ao microfone condensador cardióide, marca Shure ${ }^{\circledR}$, modelo $16 \mathrm{~A}$, situado a $10 \mathrm{~cm}$ da falante, e em ambiente silencioso com um nível de ruído ambiental inferior a 50 dBNPS verificado através do medidor de nível de pressão sonora da marca Radio Shac ${ }^{\circledR}$. O material de voz foi transferido para um computador da marca Dell ${ }^{\circledR}$, modelo Optiplex GX260, placa de som profissional marca Direct Sound $^{\circledR}$, e analisado no programa CSL, da Kay Elemetrics $₫$, no módulo Multi-Dimensional Voice Program (MDVP) em que foi extraída a frequência fundamental $\left(f_{0}\right)$ e no módulo Real Time, foi extraída a extensão fonatória máxima em semitons e as frequências máximas e mínimas em $\mathrm{Hz}$ das vozes registradas.
Este trabalho foi aprovado pelo Comitê de Ética em Pesquisa da Universidade Federal de Minas Gerais - COEP, no dia 25 de outubro de 2006, sob o parecer no ETIC 0375/06.

A análise estatística dos valores obtidos constituiu-se da obtenção dos valores da média e desvio padrão para os três grupos estudados. Para verificar a correlação entre a idade e os valores acústicos analisados foi utilizado o coeficiente de correlação de Pearson. O nível de significância adotado foi de $5 \%$, assinalando-se com um asterisco $\left({ }^{*}\right)$ os valores estatisticamente significantes.

\section{RESULTADOS}

A população que participou deste estudo foi composta por 96 idosos, do sexo feminino, com idades variando entre 60 e 103 anos, com média de 71,10 anos e desvio padrão de 8,65 , que foram distribuídas em três grupos conforme a faixa etária. 
A Tabela 1 apresenta os valores médios e o desvio-padrão da frequência fundamental em $\mathrm{Hz}$ nos três grupos estudados.

$\mathrm{Na}$ Tabela 2 encontram-se os valores médios da extensão fonatória máxima em semitons e das frequências máximas e mínimas em $\mathrm{Hz}$, assim como os valores de desvio-padrão nos três grupos estudados.

A Tabela 3 mostra os valores de média e desviopadrão do tempo máximo de fonação das vogais /a/, /i/ e /u/ nos três grupos estudados.
$\mathrm{Na}$ Tabela 4 encontram-se os valores do coeficiente de correlação de Pearson para a idade e as medidas acústicas estudadas. Observa-se que os valores da extensão fonatória, da frequência máxima e do TMF da vogal /u/ apresentaram evidências de correlação com a idade $(p<0,05)$. A vogal /a/ apresentou correlação quase significante (p próximo de 0,05 ). O sinal negativo do coeficiente de correlação de Pearson indica uma correlação negativa entre as medidas acústicas estudadas e a idade, ou seja, a aumento da idade provoca uma diminuição destas medidas.

Tabela 1 - Valores da média e desvio padrão da frequência fundamental em Hertz para os três grupos estudados

\begin{tabular}{|c|c|c|c|c|}
\hline Fo & Grupo 1 & Grupo 2 & Grupo 3 & Total \\
\hline Média & 193,81 & 195,71 & 187,60 & 193,26 \\
\hline Desvio-padrão & 24,80 & 27,30 & 21,60 & 24,94 \\
\hline
\end{tabular}

Legenda: $F_{0}$ - Frequência Fundamental

Tabela 2 - Valores da média da extensão fonatória em semitons, da frequência mínima e da frequência máxima em Hertz e seus respectivos desvio-padrão para os três grupos estudados

\begin{tabular}{lcccc}
\hline & & Semitons & Mínima & Máxima \\
\hline \multirow{2}{*}{ Grupo 1 } & Média & 42,105 & 103,62 & 1099,1 \\
& Desvio-padrão & 9,387 & 75,65 & 372,4 \\
\hline \multirow{2}{*}{ Grupo 2 } & Média & 37,29 & 102,28 & 918,1 \\
& Desvio-padrão & 10,65 & 34,87 & 435,6 \\
\hline \multirow{2}{*}{ Grupo 3 } & Média & 38,75 & 88,73 & 876,8 \\
& Desvio-padrão & 9,27 & 45,17 & 420,1 \\
\hline \multirow{2}{*}{ Total } & Média & 40,10 & 109,28 & 1006,91 \\
& Desvio-padrão & 8,45 & 89,33 & 362,13 \\
\hline
\end{tabular}

Tabela 3 - Valores da média e desvio-padrão do tempo máximo de fonação em segundos das vogais /a/, /i/ e /u/ para os três grupos estudados

\begin{tabular}{lcccc}
\hline & & Vogal /a/ & Vogal /i/ & Vogal /u/ \\
\hline \multirow{2}{*}{ Grupo 1 } & Média & 8,546 & 8,824 & 9,229 \\
& Desvio-padrão & 3,462 & 3,671 & 6,449 \\
\hline \multirow{2}{*}{ Grupo 2 } & Média & 7,975 & 8,447 & 8,094 \\
& Desvio-padrão & 3,124 & 3,980 & 3,695 \\
\hline \multirow{2}{*}{ Grupo 3 } & Média & 7,521 & 8,052 & 7,954 \\
& Desvio-padrão & 4,182 & 4,375 & 3,746 \\
\hline \multirow{2}{*}{ Total } & Média & 8,20 & 8,58 & 8,67 \\
& Desvio-padrão & 3,24 & 3,64 & 4,34 \\
\hline
\end{tabular}


Tabela 4 - Valores do coeficiente de correlação de Pearson para a idade e para os valores das medidas acústicas de frequência fundamental em $\mathrm{Hz}$, extensão fonatória em semitons, frequência máxima, frequência mínima e tempo máximo de fonação das vogais /a/, /i/e / /u/

\begin{tabular}{lccccccc}
\hline & Fo & EF & Fmax & Fmin & /a/ & /i/ & /u/ \\
\hline Coeficiente de correlação & $-0,045$ & $-0,186$ & $-0,221$ & $-0,040$ & $-0,101$ & $-0,078$ & $-0,137$ \\
Valor-P & 0,664 & $0,002^{*}$ & $0,000^{*}$ & 0,503 & 0,088 & 0,184 & 0,020 \\
\hline
\end{tabular}

Legenda: $F_{0}$ - Frequência Fundamental; EF - Extensão fonatória; Fmax - Frequência máxima; Fmin - Frequência mínima ${ }^{*} \mathrm{P}<0,05$

\section{DISCUSSÃO}

O aumento da longevidade e a redução das taxas de mortalidade, nas últimas décadas do século passado, mudaram o perfil demográfico do Brasil ${ }^{1}$.

O envelhecimento é considerado uma etapa natural do desenvolvimento sendo este processo progressivo e degenerativo, caracterizado por menor eficiência funcional ${ }^{3,4,7,8}$. O declínio de algumas das funções na senilidade reflete-se em alterações cardiovasculares, musculares, esqueléticas, neurológicas, sensoriais e respiratórias. Como a produção vocal depende do funcionamento desses sistemas, a voz poderá apresentar mudanças ${ }^{4}$.

Conhecer os parâmetros da voz no envelhecimento é fundamental para se atuar com prevenção e para se elaborar programas de reabilitação eficientes. Para uso clínico, são medidas acústicas importantes a frequência fundamental $\left(f_{0}\right)$, o perfil da extensão vocal máxima e o tempo máximo de fonação (TMF).

A frequência fundamental da voz de um indivíduo é o reflexo das características biodinâmicas das pregas vocais e de sua integração com a pressão subglótica. A extensão fonatória máxima tem relação direta com a saúde das pregas vocais e correlaciona-se com a percepção da disfonia, ou seja, quanto maior a limitação da extensão da voz, mais severa a percepção da alteração que afeta a qualidade vocal ${ }^{3}$. Já o TMF é um dos parâmetros com o qual se obtêm de modo mais prático as medidas respiratórias, com possibilidade de ser empregado tanto como um meio de diagnóstico quanto de acompanhamento e evolução de terapia em pacientes disfônicos. É uma medida acústica importante e prática na descrição do comportamento vocal, pois o falante, na fala encadeada, realiza recargas aéreas a cada um terço de seu TMF ${ }^{3}$.

Diversos estudos na literatura apontam para a diminuição da frequência fundamental na voz de mulheres idosas ${ }^{9}$. As mulheres adquirem um pitch mais grave, o que se constitui numa virilização vocal que reflete as mudanças fisiológicas que ocorrem na laringe com o aumento da idade ${ }^{3,10}$. A diminuição da $\mathrm{f}_{0}$ foi atribuída por alguns autores a um alto índice de edema da mucosa das pregas vocais, devido às alterações pós-menopausa ${ }^{11,12}$.

Um estudo realizado com 20 homens e 20 mulheres com idade média de 75 anos avaliou a prevalência de alterações laríngeas e das características perceptivo-auditivas da voz e os valores da frequência fundamental. No grupo de idosos do sexo feminino, os autores encontraram uma média de $167 \mathrm{~Hz}$ para a frequência fundamental além de uma qualidade vocal rouca ${ }^{11}$.

Outra pesquisa caracterizou os parâmetros acústicos da voz de 180 indivíduos idosos, sendo 128 mulheres com idade média de 62,35 anos. Tais parâmetros constituíram-se de medidas acústicas a curto prazo e no valor da frequência fundamental da vogal /a/. Os pesquisadores encontraram para idosas o valor de $186,95 \mathrm{~Hz}^{13}$.

Para o programa CSL- 4300 da Kay-Elemetrics $\AA$ a medida de $f_{0}$ para mulheres jovens com vozes neutras foi normatizada em um estudo com 20 indivíduos com idade entre 20 e 45 anos, encontrandose, para a vogal /a/, o valor de $205,82 \mathrm{~Hz}^{14}$.

O presente estudo, que avaliou a voz de 96 idosas, distribuídas em três grupos conforme a faixa etária encontrou valores de frequência fundamental de 193,81 Hz para o Grupo 1, 195,71 para o Grupo 2 e de $187,60 \mathrm{~Hz}$ para o Grupo 3 (Tabela 1). Tais valores estão próximos aos encontrados na literatura com mulheres idosas e menores do que os encontrados com a população jovem, apontando para a diminuição da frequência fundamental com o envelhecimento ${ }^{11-14}$. Quando se comparou os valores da frequência fundamental nos três grupos estudados não houve diferença estatística, porém, é importante salientar que o valor negativo do coeficiente de correlação de Pearson demonstra que o aumento da idade provoca uma diminuição da frequência fundamental (Tabela 4).

Falantes normais do Português Brasileiro, com idades entre 20 a 40 anos, do sexo feminino e que não fazem uso profissional da voz, apresentam extensão vocal com valor mínimo de 20 semitons e máximo de 39 semitons. A faixa de extensão vocal, 
segundo alguns autores, varia de 13 semitons a, aproximadamente, 55 semitons. Indivíduos com pregas vocais sadias devem apresentar um mínimo de 20 semitons ${ }^{15}$.

A extensão fonatória máxima tende a diminuir com o avanço da idade, de acordo com a literatura ${ }^{3,16,17}$. Para a população idosa a média da frequência mínima e máxima, respectivamente, é de $154,83 \mathrm{~Hz}$ e $478,88 \mathrm{~Hz}$. Já a média da extensão vocal encontrada é 19,70 semitons medidas pela Fonetografia ${ }^{16}$. A presbifonia é atribuída à irregularidade na vibração de pregas vocais, incompetência glótica, atrofia de pregas vocais, tensão laríngea e às alterações histológicas dos tecidos conjuntivos ${ }^{11}$. No atual estudo, a extensão fonatória apresentouse com valores semelhantes aos encontrados em adultos jovens, conforme a literatura relata ${ }^{8}$. Os valores encontrados para a média da frequência mínima e máxima foram, respectivamente, para o Grupo 1 de 103,62 Hz e 1099,1 Hz; para o Grupo 2 de $102,28 \mathrm{~Hz}$ e $918,10 \mathrm{~Hz}$ e para o Grupo 3 de $88,73 \mathrm{~Hz}$ e $876,8 \mathrm{~Hz}$. Já a média da extensão fonatória máxima encontrada foi de 42,10 semitons para o Grupo 1; 37,29 semitons para o Grupo 2 e 38,75 semitons para o Grupo 3 (Tabela 2). Apesar dos valores se aproximarem aos referidos na literatura para jovens, houve diferença estatisticamente significante entre os grupos para os valores de extensão fonatória e de frequência máxima (Tabela 4). Tais resultados sugerem que quanto mais idosa a mulher menor sua capacidade de variação de frequência, principalmente, para a região mais aguda da extensão vocal, o que concorda com a literatura que aponta que a extensão fonatória máxima tende a diminuir com o avanço da idade $3,8,10$.

Os valores da extensão fonatória máxima estarem próximos aos apontados para indivíduos jovens pela literatura pode ser explicado pelo fato de que neste estudo, as vozes analisadas eram de indivíduos do sexo feminino sem queixa vocal e que não apresentavam alteração laríngea e/ou haviam realizado cirurgias laríngeas. Deve-se salientar que os indivíduos com boas condições físicas têm menos alterações vocais do que aqueles do mesmo grupo etário com piores condições de saúde ${ }^{4}$. Além disso, deve-se levar em consideração a forma de extração de tais medidas, que no caso da presente pesquisa configuraram-se na extração automática das mesmas pelo programa CSL e, não medidas obtidas pela Fonetografia.

Alterações estruturais degenerativas decorrentes do envelhecimento, em especial modificações laríngeas, ocasionam menor elasticidade e força das pregas vocais, observando-se frequentemente a presença de fenda glótica associada com atrofia e o decréscimo na massa com hiperconstrição supraglótica compensatória ${ }^{11}$ e, além disso, observa-se a presença do arqueamento das pregas vocais 4,5 . Indivíduos com fendas glóticas apresentam TMF diminuídos, o que gera a formação de frases mais curtas e dificulta o processo comunicativo ${ }^{3}$.

O TMF, de acordo com a literatura, é reduzido no idoso em relação ao adulto jovem em decorrência das modificações laríngeas no envelhecimento $3,4,6,7,10,12,18,19$.

O valor médio de TMF, encontrado na literatura, para a população idosa, oscila em decorrência do número de indivíduos avaliados e em relação ao tipo de vogal utilizada para a avaliação. Uma pesquisa que mediu o TMF de 10 mulheres e dois homens com idade entre 54 e 74 anos com o objetivo de verificar a efetividade de um programa de reabilitação vocal para idosos, encontrou o valor da medida do TMF da vogal /a/ de 14,4 segundos no momento pré e 17,5 segundos no pós-programa vocal ${ }^{19}$. Em outro estudo, que avaliou 25 idosos de 62 a 86 anos encontrou valores de TMF em segundos, no momento pré-intervenção fonoaudiológica de 12; 12,1 e 12,2 segundos para as vogais /a/, /i/ e /u/, respectivamente, e de 13,3; 13,8 e 13,4 segundos no momento pós-intervenção, para as mesmas vogais ${ }^{18}$. No presente estudo, a média encontrada para o TMF da vogal /a/ no Grupo 1 foi de 8,54 segundos, no Grupo 2 de 7,97 segundos e no Grupo 3 de 7,52 segundos. Para a vogal /i/ no Grupo 1 o valor encontrado foi de 8,82 segundos, no Grupo 2 de 8,44 segundos e no Grupo 3 de 8,05 segundos. Para a vogal /u/ os valores do TMF do Grupo 1 foram de 9,22 segundos, no Grupo 2 de 8,09 segundos e no Grupo 3 de 7,95 segundos (Tabela 3), concordando, portanto, com os trabaIhos que afirmam que o TMF diminui com o enveIhecimento $3,4,6,7,10,12,18,19$.

Foi encontrada diferença estatisticamente significante entre os grupos e os valores dos TMF apenas para a vogal /u/, porém, a presença do coeficiente de correlação de Pearson com valores negativos em todas as vogais demonstra que com o aumento da idade há uma tendência de diminuição dos valores do TMF (Tabela 4). Vale ressaltar que como os valores do coeficiente de correlação de Pearson são baixos, a influência da idade sobre estas medidas é moderada, indicando que a mudança é lenta com o avanço da idade.

A melhor compreensão dos aspectos acústicos da voz em indivíduos idosos se faz necessária para melhor compreender a dinâmica vocal e laríngea neste grupo de indivíduos, aprimorando os aspectos de promoção e intervenção fonoaudiológica da voz. 


\section{CONCLUSÂO}

Analisando acusticamente a voz de idosas, podese concluir que há uma diminuição da frequência fundamental e do tempo máximo de fonação com o envelhecimento. Os valores da extensão fonatória máxima e da frequência mínima e máxima foram semelhantes aos de adultos jovens. Os valores da extensão fonatória máxima, da frequência máxima e do TMF da vogal /u/ mostraram diferenças estatisticamente significantes entre os diferentes grupos de idade. Todas as medidas acústicas estudadas mostraram uma tendência de diminuição com o aumento da idade.

\begin{abstract}
Purpose: to analyze acoustic measures in elderly women's voices. Methods: ninth six elderly women, with ages between 60 and 103-year old, were recruited and separated in tree groups according to their ages. Results: the values of fundamental frequency were 193. $81 \mathrm{~Hz}$ (Group 1), 195.71 (Group 2) and $187.60 \mathrm{~Hz}$ (Group 3). The Maximum phonatory extension were 42.10 semitones (Group 1); 37.29 semitones (Group 2) and 38.75 semitones (Group 3). The values of the minimum frequency and maximum frequency were respectively $103.62 \mathrm{~Hz}$ and $1099.1 \mathrm{~Hz}$ (Group 1); $102.28 \mathrm{~Hz}$ and 918.10 $\mathrm{Hz}$ (Group 2) and $88.73 \mathrm{~Hz}$ and $876.8 \mathrm{~Hz}$ (Group 3). The maximum phonation time of the vowel /a/ were 8.54 seconds (Group 1), 7.97 seconds (Group 2) and 7.52 seconds (Group 3). For the vowel /i/ the values were 8.82 seconds (Group 1), 8.44 seconds (Group 2) and 8.05 seconds (Group 3). For the vowel /u/ were 9.22 seconds (Group 1), 8.09 seconds (Group 2) and 7.95 seconds (Group 3). Conclusion: elderly women showed lower values of fundamental frequency and maximum phonation time. Maximum phonatory extension measure was similar to those found in young adults. All studied measures showed a decreasing trend with advancing age.
\end{abstract}

KEYWORDS: Voice; Aging; Speech Acoustics

\section{REFERÊNCIAS}

1. Lima-Costa MF, Barreto SM, Giatti L. Condições de saúde, capacidade funcional, uso de serviços de saúde e gastos com medicamentos da população idosa brasileira: um estudo descritivo baseado na Pesquisa Nacional por Amostra de Domicílio. Cad Saúde Publ. 2003; 19(3):735-43.

2. Prado SD, Sayd JD. A pesquisa sobre envelhecimento humano no Brasil: grupos e linhas de pesquisa. Ciência \& Saúde Coletiva. 2004; 9(1):57-67.

3. Behlau MS. Voz: o livro do especialista. vol 2. Rio de Janeiro: Revinter; 2005.

4. Pontes P, Brasolotto A, Behlau M. Glottic characteristics and voice complaint in the elderly. J Voice. 2005; 19(1):84-94.

5. Costa HO, Matias C. O impacto da voz na qualidade da vida da mulher idosa. Rev Bras Otorrinolaringol. 2005; 71(2):172-8.

6. Polido AM, Martins MASUR, Hanayama EM. Percepção do envelhecimento vocal na terceira idade. Rev CEFAC. 2005; 7(2):241-51.

7. Menezes LN, Vicente LCC. Envelhecimento vocal em idosos institucionalizados. Rev CEFAC. 2007; 9(1):90-8.
8. Baraldi GS, Almeida LC, Calais LL, Borges ACC, Gielow I, Cunto MR. Estudo da frequência fundamental da voz de idosas portadoras de diferentes graus de perda auditiva. Rev Bras Otorrinolaringol. 2007; 73(3):378-83.

9. Santos IR, Pereira JC. Caracterização dos parâmetros acústicos da voz na terceira idade. In: IV Encontro de Bioengenharia da USP, 2004, São Carlos (SP): Universidade São Paulo; 2004.

10. Feijó A, Estrela F, Scalco M. Avaliação perceptiva e quantitativa da voz na terceira idade. Fonoaudiol Brasil. 1998; 1(1):22-31.

11. Honjo I, Isshiki N. Laryngoscopic and voice characteristics of aged persons. Arch Otolaryngol. 1980; 106(3):149-50.

12. Morsomme D. Presbyphonia: voice differences between the sexes in the elderly: comparison by maximum phonation time, phonation quotient and spectral analysis. Logop Phoniatr Vocol. 1997; 22(1):9-14.

13. Santos IR. Análise acústica da voz de indivíduos na terceira idade. [dissertação]. São Paulo (SP): Universidade de São Paulo; 2005. 
14. Felippe ACN, Grillo MHMM, Grechi TH. Normatização de medidas acústicas para vozes normais. Rev Bras Otorrinolaringol. 2006; 72(5):659-64.

15. Vargas AC, Costa AG, Hanayama EM. Perfil da extensão vocal em indivíduos falantes normais do português brasileiro. Rev CEFAC. 2005; 7(1):108-16.

16. Teles-Magalhães LC, Pegoraro-Krook MI, Pegoraro R. Study of the elderly females' voice by phonetography. J Voice. 2000; 14(3):310-21.
17. Rocha TF, Amaral FP, Hanayama EM. Extensão vocal de idosos coralistas e não coralistas. Rev CEFAC. 2007; 9(2):248-54.

18. Venites JP, Bertachini L, Ramos LR. Atuação fonoaudiológica na presbifonia: a efetividade de uma proposta terapêutica. Rev Fonoaudiol Brasil. 2004; 4(1):1-8.

19. Monte CA, Mourão LF, Mota PHM. Avaliação fonoaudiológica e otorrinolaringológica em idosos pré e pós-programa vocal. Pró-Fono. 2001; 13(2): 169-76.

DOI: 101590 / S1516 - 18462009005000017

RECEBIDO EM: 31/12/2007

ACEITO EM: 22/09/2008

Endereço para correspondência:

Ana Cristina Côrtes Gama

Av Professor Alfredo Balena, 190 sala 69

Belo Horizonte - MG

CEP: $30130-100$

E-mail: anacgama@medicina.ufmg.br; anaccgama@gmail.com 